\title{
"PLASMA ACCELERATION ON MULTISCALE TEMPORAL VARIATIONS OF ELECTRIC AND MAGNETIC FIELDS DURING SUBSTORM DIPOLARIZATION IN THE EARTH'S MAGNETOTAIL,
}

\author{
Elena Igorevna Parkhomenko ${ }^{1}$, Helmi Vitalevna Malova ${ }^{2,1, *}$, Elena Evgenevna \\ Grigorenko ${ }^{1}$, Victor Yurevich Popov ${ }^{4,3,1}$, Anatolii Alekseevich Petrukovich ${ }^{1}$, \\ Dominique C. Delcourt ${ }^{5}$, Elena Aleksandrovna Kronberg ${ }^{6,7}$, Patric W. Daly ${ }^{6}$, \\ Lev Matveevich Zelenyi ${ }^{1}$
}

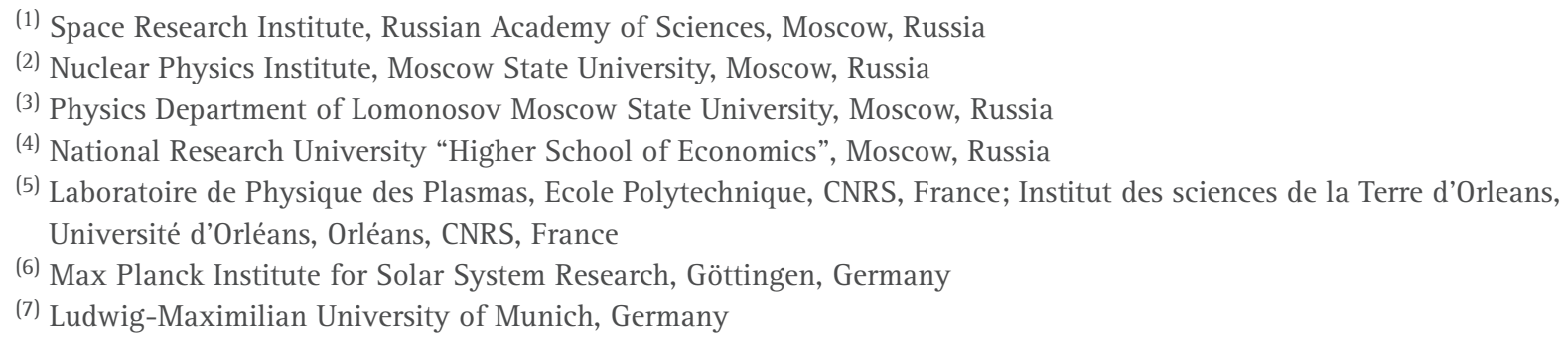

Article history

Receveid November 9, 2017; accepted March 15, 2018.

Subject classification:

Magnetotail; Dipolarization; Electric field fluctuations; Particle acceleration; Numerical modeling.

\begin{abstract}
Magnetic field dipolarizations are often observed in the magnetotail during substorms. These generally include three temporal scales: (1) actual dipolarization when the normal magnetic field changes during several minutes from minimum to maximum level; (2) sharp $B_{z}$ bursts (pulses) interpreted as the passage of multiple dipolarization fronts with characteristic time scales $<1$ min, and (3) bursts of electric and magnetic fluctuations with frequencies up to electron gyrofrequency occurring at the smallest time scales ( $\leq 1 \mathrm{~s}$ ). We present a numerical model where the contributions of the above processes (1)-(3) in particle acceleration are analyzed. It is shown that these processes have a resonant character at different temporal scales. While $0^{+}$ions are more likely accelerated due to the mechanism (1), $\mathrm{H}^{+}$ ions (and to some extent electrons) are effectively accelerated due to the second mechanism. High-frequency electric and magnetic fluctuations accompanying magnetic dipolarization as in (3) are also found to efficiently accelerate electrons.
\end{abstract}

\section{INTRODUCTION}

A number of observational and theoretical investigations have revealed the importance of accelerated particle flows in the Earth's magnetotail [Sharma et al., 2008; Retino et al., 2008; Yamada et al., 2010; Fu et al., 2011; Runov et al., 2011a; Birn et al., 2012, 2013; Ashour-Abdalla et al., 2015; Grigorenko et al., 2015], that conversely point to specific regions for energy conversion during substorms [Zelenyi et al., 2008; Zelenyi et al., 2011 and references therein; Angelopoulos et al., 2013]. Thanks to Geotail, Cluster, THEMIS, MMS and other spacecraft observations in the Earth's environment, significant information on the acceleration mech- anisms has been collected [e.g., Artemyev et al., 2012; Lui, 2014 , Runov et al., 2014; Grigorenko, 2015; Kronberg et al., 2017; Liang et al., 2017] but details of these mechanisms are not completely understood up to the present day. Some satellite missions demonstrated the presence of particles with energies up to hundreds of $\mathrm{keV}$ in the Earth's magnetotail [e.g., Zhou et al., 2010; Ashour-Abdalla et al., 2015; Kronberg et al., 2017] but their origin and the processes at work remain unclear, although studies during the last decades [Baker et al., 1985, Zelenyi et al., 2011; Grigorenko et al., 2011; Angelopoulos et al., 2013, and references there in] have made clearer the role of magnetotail current sheet evolution in the energy conversion during substorms [Birn 
et al., 2012; Lui, 2014].

During the growth phase of substorms, magnetic flux in the magnetospheric lobes is increased, leading to thinning of the magnetotail current sheet from a thickness of 1 to $2 \mathrm{RE}$ down to a thickness of a few ion gyroradii (250-2000 km) [Sergeev et al., 1993; Sergeev et al., 1996; Runov et al., 2008, Nakamura et al., 2008]. Such a thin current sheet (TCS) configuration has been shown to be metastable and can be a reservoir of free magnetic energy that is explosively released during current sheet destruction [Galeev, 1979; Baker et al., 1985; Zelenyi et al., 1990; Zelenyi et al., 2008]. Later phases of substorms are characterized by abrupt changes of magnetotail magnetic field and earthward propagation of Dipolarization Fronts (DF) [Runov et al., 2009, 2011, 2012; Yao et al., 2016] that are step-like structures with sharp enhancements of the normal magnetic field $B_{z}$ [e.g., Nakamura et al., 2002; Runov et al., 2009; Sergeev et al., 2009] (note that GSM coordinate system is used in the present study). These fronts are often associated with an enhancement of plasma and magnetic pressure as well as Bursty Bulk Flows (BBF) that are accelerated earthward [e.g. Angelopoulos et al., 1992; Sergeev et al., 2011; Runov et al., 2011; Fu et al., 2011]. DFs typically appear in the midtail region after substorm onsets [Lui, 2014] and have a tendency to slow down and to thicken during their motion toward the Earth [Hamrin et al., 2013 ]. Several mechanisms have been proposed to explain the generation of DFs: (1) BBF-type flux ropes [Slavin et al., 2003], (2) nightside flux transfer events [Sergeev et al., 1992], (3) generation of burstlike magnetic structures by impulsive magnetic reconnection in the magnetotail [Heyn and Semenov, 1996; Semenov et al., 2005; Longcope and Priest, 2007, Sitnov et al., 2009; Sitnov and Swisdak, 2011]. Evidences of such DFs have been found in a variety of spacecraft observations [e.g., Nakamura et al., 2002; Sharma et al., 2008; Runov et al., 2009] and their relation with reconnection processes is now clearly established. Acceleration of charged particles as a result of magnetic reconnection is now considered as one of the most effective mechanisms to accelerate particles to high energies [Yamada et al., 2010].

Furthermore, in a number of instances, the passage of DFs is accompanied by strong plasma turbulence or electrostatic fluctuations [Ono et al., 2009; El-Alaoui et al., 2013; Lui, 2014; Grigorenko et al., 2016] and by plasma acceleration [Zhou et al., 2010; Fu et al., 2011; Artemyev et al., 2012; Birn et al., 2012, 2013; Grigorenko et al., 2011, 2015, 2017]. Ions with energies about a few hundreds of keV have been frequently observed during substorm dipolarization in the near-Earth tail [e.g., Ipavich et al., 1984; Nosé et al., 2000; Ono et al., 2009; Keika et al., 2010] or in the presence of turbulent electromagnetic field in the plasma sheet [Cattell and Mozer, 1982; Hoshino et al., 1994; Bauer at al., 1995, Ono et al., 2009]. Because DFs travel over large distances from the middle (or deep) magnetotail toward the planet without significant evolution [Runov et al., 2009], particle acceleration in the course of the interaction with DFs can be effective due to the long time dynamics of such fronts.

Generally several mechanisms responsible for particle acceleration or heating by DFs have been proposed:

1) Nonadiabatic (in the sense of violation of the first adiabatic invariant) plasma acceleration by the inductive electric field that results from the magnetic field reconfiguration [Delcourt et al., 1990; Veltri et al., 1998; Delcourt, 2002; Ono et al., 2009; Greco et al., 2015 ]. Works by [Perri et al., 2009; Greco et al., 2009; Ono et al., 2009] also demonstrated the importance of nonadiabatic acceleration of $\mathrm{H}+$ ions in the course of their resonant interaction with the low-frequency magnetic fluctuations in the region behind the dipolarization front.

2) adiabatic energy gain of magnetized electrons and ions due to local increase of the magnetic field [Delcourt et al., 1990; Birn et al., 2004; Apatenkov et al., 2007; Fu et al., 2011; Birn et al., 2012];

3) Particle acceleration near the magnetic reconnection site by the cross-tail electrostatic field $E_{y}$ [e.g., Hoshino, 2005; Retino et al., 2008];

4) Reflection from fronts [Zhou et al., 2010, 2012] and resonant capture by fronts [Artemyev et al., 2012b; Ukhorskiy et al., 2017] can also lead to nonadiabatic gain of energy because DF thickness is comparable to the gyroradii of hot ions.

5) Particle pick-up in outflows near reconnection regions and consequent heating up to thermal velocities of the order of the Alfvén speed [Drake et al., 2009].

From a theoretical viewpoint, most of these mechanisms have been investigated with help of numerical simulations in model magnetotail current sheets, and estimates of their efficiency have been provided in different studies [e.g., Zelenyi et al., 1990; Veltri et al., 1998; Zelenyi et al., 2008; Greco et al., 2009; Artemyev et al., 2012, Greco et al., 2015]. Acceleration by the dawn-dusk electrostatic potential drop is clearly limited in efficiency and can reach maximum energies about $30-90 \mathrm{keV}$ in the potential drop of about $50-70 \mathrm{kV}$ across the magnetotail [Grigorenko et al., 2009]. On the other hand, electromagnetic turbulence can provide sev- 
eral orders of magnitude in particle energy increase, but this effect was only investigated in the distant (about $100 R_{E}$ ) magnetotail where the normal magnetic field component becomes weak and stochastic [Perri et al., 2008; Zelenyi et al., 2008, 2011]. Acceleration of heavy ions due to plasma turbulence has also been investigated in [Greco et al., 2015; Grigorenko et al., 2015; Catapano et al., 2016; Liang et al., 2017]. All the above mentioned works put forward a spatial resonance character between particles and fields, that depends upon the Larmor radius and the nonstationary structures of the magnetic field.

The purpose of the present work is to put into perspective the combination of the different processes occurring during magnetic dipolarization, from (1) local large-scale dipolarization including (2) multiple front passages and (3) subsequent high-frequency electromagnetic oscillations with $f \leq f_{\text {ce }}\left(f_{\text {ce }}\right.$ being the electron cyclotron frequency). These latter oscillations that are observed during the late stage of dipolarization have been shown to contribute to electron energization [Grigorenko et al., 2016]. In the following, we will emphasize the multiscale temporal character of the overall dipolarization process (1)-(3) and the distinct resonant contributions to particle acceleration. This acceleration will be investigated using a simple numerical model taking into account different time scales, a temporal analog of the well known Russian "matreshka" consisting of many dolls embedded one into the other as it is shown in Figure 1. More specifically, to reconstruct the above mentioned (1)-(3) processes during magnetic dipolarization, three different time scales were considered in the present study. We will compare the numerical results obtained with in situ measurements, and will discuss the role of the acceleration mechanisms on different temporal scales.

\section{NUMERICAL MODEL}

Test particle simulations performed to examine the particle dynamics in the magnetotail configuration $\mathrm{B}_{0}$ in the presence of dipolarization processes $\Delta B(t)$ followed by high frequency fluctuating electric field $\delta \mathrm{E}(\mathrm{r}, t)$ and related induced magnetic field $\delta \mathrm{B}(\mathrm{r}, t)$. The time varying magnetic field is considered to be a superposition of the following components:

$$
\mathbf{B}(\mathrm{r}, t)=\mathbf{B}_{0}(\mathrm{z})+\Delta \mathbf{B}(t)+\delta \mathrm{B}(\mathbf{r}, t)
$$

where $B_{0}(z)$ is the initial field in the magnetotail current sheet before the dipolarization onset, $\Delta \mathrm{B}(t)=\Delta\left\{\mathrm{B}_{\mathrm{d}}(t)+\mathrm{B}_{\mathrm{DF}}(t)\right\}$ is the time-dependent magnetic field, which comprises both the general

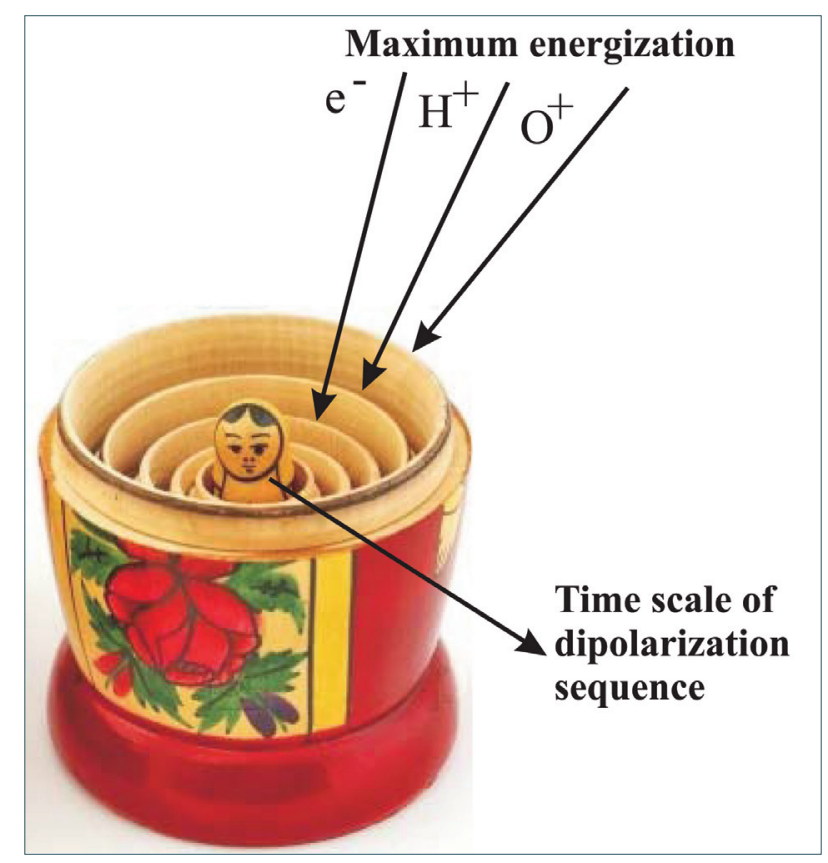

FIGURE 1. Illustration of multiscale embedded property of time scales during geomagnetic dipolarization and the resonance character of particle acceleration, where the maximum energization is achieved when the scales of magnetic disturbances are about particles gyroperiods.

dipolarization $\Delta \mathrm{B}_{\mathrm{d}}(t)$ and the multiple $\mathrm{DFs} \Delta \mathrm{B}_{\mathrm{DF}}(t)$ arriving to the observer at distinct times $t ; \delta \mathrm{B}(\mathrm{r}, t)$ is the amplitude of the induced fluctuating magnetic component.

The magnetotail field is taken in the form of the Harris solution [Harris, 1962; Lembege and Pellat, 1982] for a tangential magnetic component $B_{x}(z)$ supplemented by a nonzero normal component of the magnetic field $B_{z}(t)$. At $t=0$, its value is equal to $B_{z 0}$ :

$$
B_{x}=B_{x 0} \cdot \tanh \left(\frac{z}{L}\right), B_{y}=0, B_{z}=B_{z 0}
$$

The electric field is taken as:

$$
\mathbf{E}(\mathbf{r}, t)=\mathbf{E}_{0}+\Delta \mathbf{E}(\mathbf{r}, t)+\delta \mathbf{E}(\mathbf{r}, t)
$$

where the large-scale dawn to dusk electric field $\mathrm{E}_{0}=E_{y} \mathrm{e}_{\mathrm{y}}$ is considered constant in the modeling region, the components of electric field $\Delta \mathrm{E}(\mathrm{r}, t)$ were found by means the Maxwell equations:

$$
\begin{gathered}
\nabla \times \Delta \mathbf{E}=-\frac{1}{c} \frac{\partial \Delta \mathbf{B}}{\partial t} \\
\nabla \cdot \Delta \mathbf{E}=0
\end{gathered}
$$

while $\delta \mathrm{E}(\mathrm{r}, t)$ is the fluctuating electric field component. As for the second and third terms in (1), they were taken from adhoc Cluster observations of $|\mathrm{B}|, B_{x}, B_{y}, B_{z}$ on July 20, 2013 (from 01:33:08 to 01:48:11 UT). As for the 
third term in (3), it was considered as a set of plane waves $\delta \mathrm{E}(\mathrm{r}, t)$ as proposed earlier in [Veltri et al., 1998; Greco et al., 2009; Perri et al., 2009 ]:

$$
\begin{gathered}
\partial E_{\mathrm{x}}=\sum_{\mathrm{k}} \partial E(\mathbf{k}) \frac{k_{\perp}}{k} g_{k}(\mathbf{r}, t) \\
\partial E_{\mathrm{y}}=\sum_{\mathrm{k}} \partial E(\mathbf{k}) \frac{k_{y} k_{x}}{k_{\perp} k} g_{k}(\mathbf{r}, t)+\frac{k_{z}}{k_{\perp}} h_{k}(\mathbf{r}, t) \\
\partial E_{\mathrm{z}}=\sum_{\mathrm{k}} \partial E(\mathbf{k}) \frac{-k_{z} k_{x}}{k_{\perp} k} g_{k}(\mathbf{r}, t)-\frac{k_{y}}{k_{\perp}} h_{k}(\mathbf{r}, t)
\end{gathered}
$$

Here $g_{\mathrm{k}}=\cos \left(\mathrm{kr}+\varphi_{\mathbf{k}}^{1}-t \omega_{\mathbf{k}}\right) \mathrm{h}_{\mathrm{k}}=\sin \left(\mathrm{kr}+\varphi_{\mathbf{K}}^{2}-t \omega_{\mathbf{k}}\right)$; $\mathrm{k}_{\perp}=\sqrt{\boldsymbol{k}_{z}^{2}+\boldsymbol{k}_{y}^{2}}, \mathrm{k}=\sqrt{\boldsymbol{k}_{z}^{2}+\boldsymbol{k}_{y}^{2}}, \mathrm{k}=\sqrt{\boldsymbol{k}_{x}^{2}+\boldsymbol{k}_{y}^{2}+\boldsymbol{k}_{z}^{2}}$. Initial phases $\varphi_{\mathbf{K}}^{1}, \varphi_{\mathbf{K}}^{2}$ are chosen randomly with uniform distribution over the interval $[0,2 \pi]$. In the present simulations, five hundred harmonics were launched into the system. Also, we chose the frequency $\omega_{\mathbf{k}}$ and amplitude $\delta E(\mathbf{k})$ of each harmonic consistently with Cluster data (recorded by C4) on July 20, 2013.

The components of the fluctuating magnetic fields $\delta B_{\mathrm{x}}, \delta B_{\mathrm{y}}, \delta B_{\mathrm{z}}$ can be obtained from Maxwell equations (assuming quasi-neutrality of plasma and the absence of free charges):

$$
\begin{gathered}
\nabla \times \partial \mathbf{B}=\frac{1}{c} \frac{\partial \quad \mathbf{E}}{\partial t} \\
\nabla \cdot \partial \mathbf{B}=0
\end{gathered}
$$

In our simulations, $5 \cdot 10^{5}$ particles were injected near the neutral plane and their equations of motion were numerically integrated inside a box having the following 3-D boundaries: $z \in\left[-L_{z}, L_{z}\right]\left(L_{z}=2 \cdot 10^{3} \mathrm{~km}\right)$; $x \in\left[-L_{x}, L_{x}\right]$ and $y \in\left[-L_{y}, L_{y}\right]$, where $L_{x}=7.5 \cdot 10^{4} \mathrm{~km}$; $L_{y}=2 \cdot L_{x}=15 \cdot 10^{4} \mathrm{~km}$. The thickness $L_{z}$ of the current sheet was set equal to $L_{z}=2000 \mathrm{~km}$, which is comparable to the proton Larmor radius (note that all quantities are normalized to the proton characteristics). When test particles leave the simulation box, their final energy is recorded to reconstruct energy distributions of plasma in the investigated region.

The initial particle velocity distribution has the form of a shifted kappa distribution that is typical for the plasma sheet of the Earth's magnetotail, viz.,

$$
\mathrm{f}(\mathbf{v})=\frac{n_{0} A_{\mathrm{k} \varepsilon}}{2\left(\sqrt{\pi k} V_{\mathrm{k} \varepsilon}\right)^{3}} \cdot \sum_{\mathrm{S}=1,2} \mathbf{1}+\frac{\mathbf{v}_{\perp}^{2}+\left(\mathbf{v}_{\mathrm{II}}-(-1)^{\mathrm{s}} \mathbf{v}_{\mathrm{D}}\right)^{2-\left(\kappa_{\varepsilon}+1\right)}}{\kappa_{\varepsilon} \cdot v_{\kappa \varepsilon}^{2}}
$$

Here $n_{0}$ is the plasma density; $v_{\kappa \varepsilon}=v_{T}$ is the thermal velocity; $\kappa_{\varepsilon}=3$ was chosen to make the distribution (6) in accordance with the real one; parameter $v_{D}=1400$ $\mathrm{km} / \mathrm{s}$ is the macroscopic characteristic of the initial distribution (6), taken constant within the simulation box.

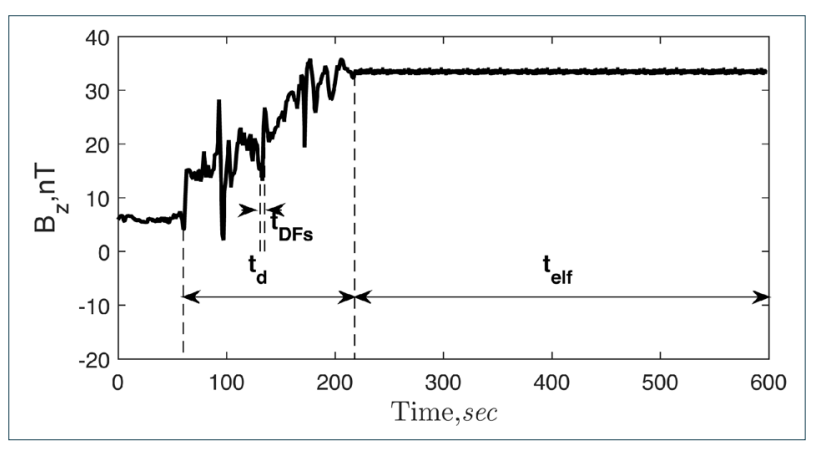

FIGURE 2. Combined profile of dipolarization $\Delta \mathrm{B}$ over time interval $\Delta t_{d}=2.6 \mathrm{~min}$, which includes multiple DFs observed at time scale $t_{D F s}$. The dipolarization is followed by the high-frequency magnetic fluctuations observed during the interval $t_{\text {elf }}=6.3 \mathrm{~min}$.

The average thermal energies $\overline{\mathrm{E}}$ of $\mathrm{e}^{-}, \mathrm{H}^{+}$and $\mathrm{O}^{+}$were chosen to be consistent with Cluster data on July 20, 2013 (from 01:33:08 to 01:48:11 UT) and equal to $\overline{\mathrm{E}}=1 \mathrm{keV}, 6 \mathrm{keV}$ and $12 \mathrm{keV}$, respectively. Typical values of the electric and magnetic field in the Earth magnetotail were taken as: $B_{z 0}=4 \mathrm{nT}, B_{0}=20 \mathrm{nT}$ and $E_{y}=0.2 \mathrm{mV} / \mathrm{m}$. The frequencies $\omega_{\mathrm{k}}$ and waves amplitude $\delta E(\mathrm{k})$ are taken for each harmonic according to the data of Cluster observations; $k$ are distributed in the range $2 \pi / L \cdot[0.05,4]$. The combined profile of magnetic field perturbations considered in the model is shown in Figure 2. Here two time intervals from $t=0$ to $t_{1}=64 \mathrm{~s}$ and from $t_{1}=64 \mathrm{~s}$ to $t_{2}=220 \mathrm{~s}$ describe correspondingly the quiet period and period of multiple dipolarizations during the time interval $t_{d}=2.6 \mathrm{~min}$ in the magnetotail observed by Clusters on July 20, 2013; the later time interval demonstrates $t_{\text {elf }}>220$ s magnetic field fluctuations on electron gyroperiod scale, calculated accordingly equation (4). In this figure, the onset of strong $B_{z}$ increase $\left(t_{0}=60 \mathrm{~s}\right)$ is marked by a vertical dashed line. During $\sim 2.6$ min (until $t_{1} \approx 220 \mathrm{~s}$ ), about 10 short intervals of large $B_{z}$ variations are observed with the average duration of about 20-30 s. At the end of dipolarization, the high-frequency electric and magnetic fluctuations with $f \leq f_{\text {ce }}$ are observed. In the following, we examine the maximum particle energies $E_{\max }$ and average energies $\bar{E}$ gained during this event, and we analyze the evolution of the spectral index $\gamma$ to characterize the acceleration mechanisms.

\section{SIMULATION RESULTS}

Figure 3 shows selected trajectories of a test proton (in blue), a test oxygen ion (in red), and a test electron (in grey) during the magnetic field dipolarization shown 

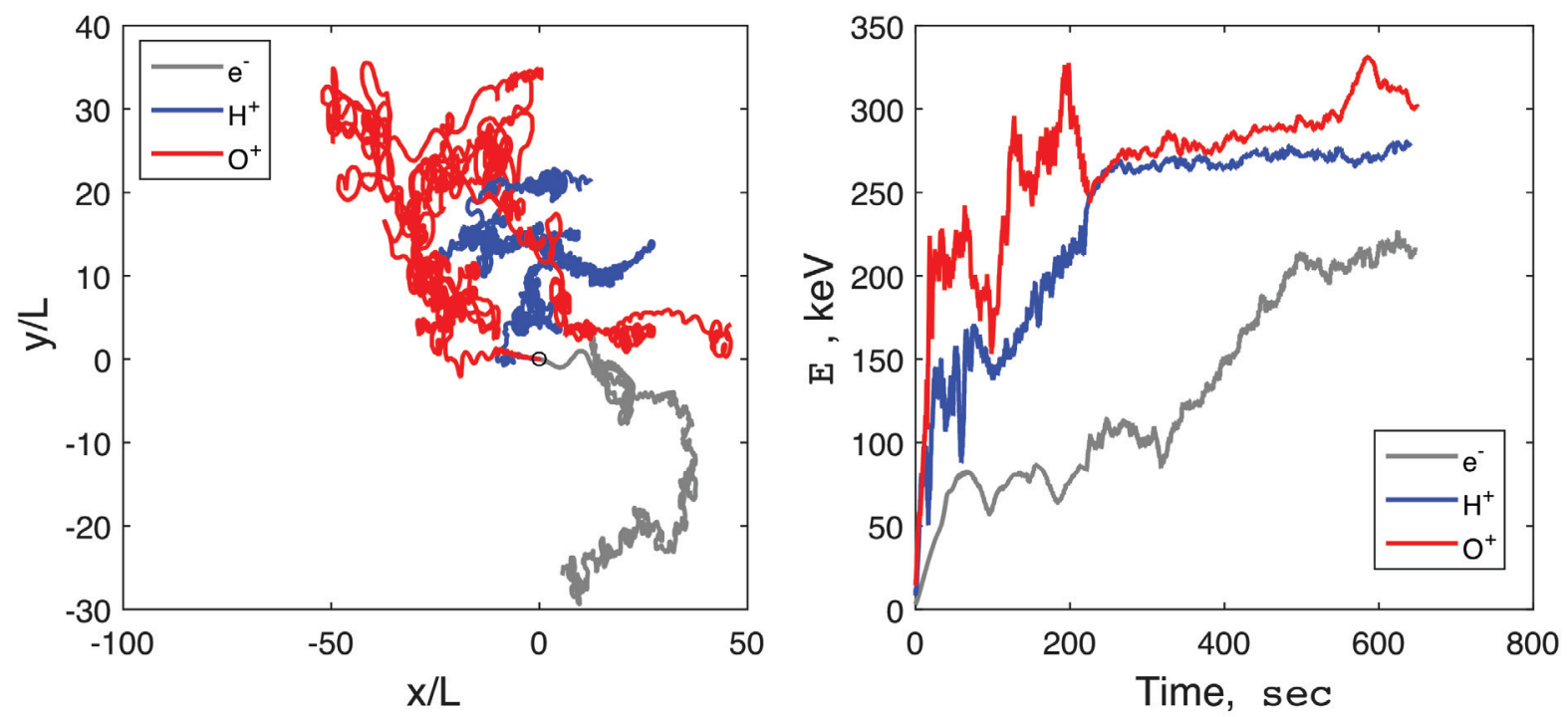

FIGURE 3. (a) Model trajectories in xy plane and (b) energy variations as a function of time for test electron (coded in grey), proton (coded in blue) and oxygen ion (coded in red) in the model magnetic field variation shown in Figure 1.

in Figure 2. It is apparent from Figure $3 \mathrm{~b}$ that, during the time interval preceding $t=220$ s, protons and oxygen ions can be accelerated by DFs from initial energies of $6 \mathrm{keV}$ and $12 \mathrm{keV}$ up to about $250 \mathrm{keV}$ and 350 $\mathrm{keV}$, respectively. During the same time interval electrons experience a strong acceleration from $1 \mathrm{keV}$ and up to $\sim 100 \mathrm{keV}$. Note also that the particle acceleration consists of sequences of energy gains and losses during the successive dipolarization fronts. After $t=220 \mathrm{~s}$, when the high-frequency electromagnetic turbulence started to be observed. Figure 3a,b displays different particle behaviors. At this time protons and oxygen ions do not experience any significant acceleration, while electrons are effectively accelerated from $\sim 100 \mathrm{keV}$ up to the $200-250 \mathrm{keV}$. This clearly demonstrates that different plasma particles may be effectively accelerated during different periods and on multiple temporal scales. While protons and oxygen ions are essentially affected by the induced electric field in the course of DFs, the most prominent electron acceleration occurs after DFs in conjunction with the electric and magnetic fluctuations.

Figure 4 presents the energy spectra obtained for the different plasma populations, $\mathrm{H}^{+}, \mathrm{O}^{+}$and $\mathrm{e}^{-}$, and for three distinct cases of acceleration, viz., passage of a single dipolarization only (case A), passage of a general dipolarization with small-scaled DFs (case B), and passage of a general dipolarization with DFs accompanied by electromagnetic turbulence (case C). Particle acceleration manifests in itself as the decrease in the spectral indices. The dependence of particle spectra upon the various time scales of the acceleration processes is also clearly noticeable in this figure. For instance, Figure 4a shows that acceleration by a single dipolarization mech-
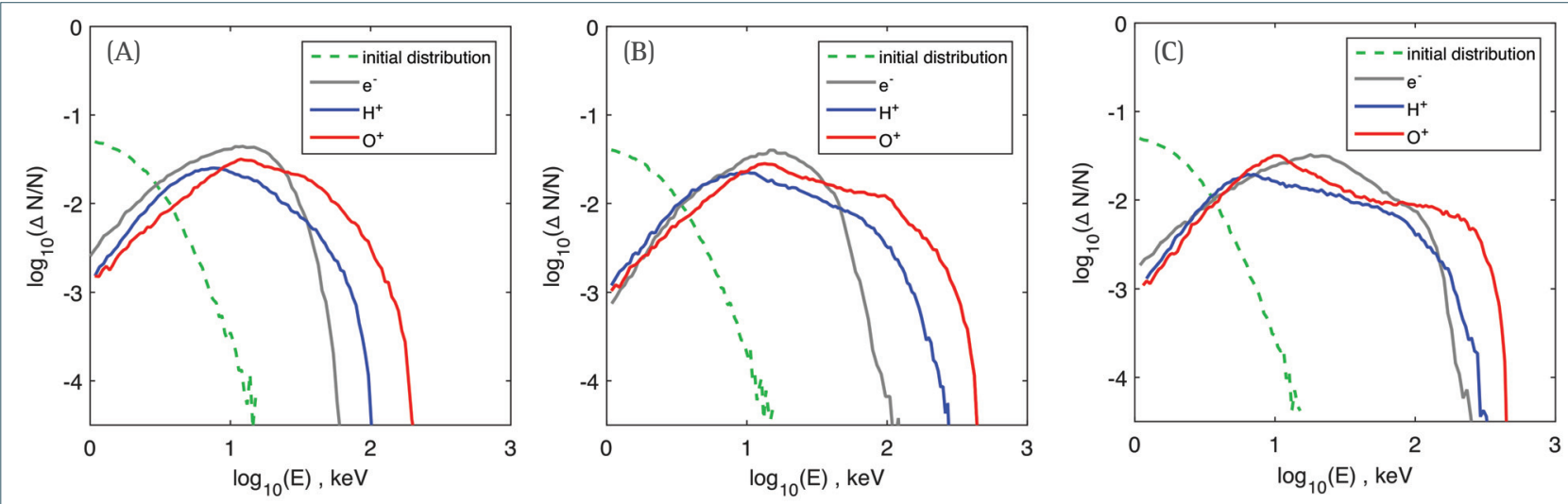

FIGURE 4. Energy spectra of $\mathrm{e}^{-}, \mathrm{H}^{+}, \mathrm{O}^{+}$in the case of: (A) single dipolarization; (B) dipolarization with DF bursts; (C) dipolarization with DF bursts followed by electric and magnetic fluctuations. 


\begin{tabular}{|c|c|c|c|c|c|c|c|}
\hline $\begin{array}{l}\text { Acceleration } \\
\text { process }\end{array}$ & Particles & $\overline{\mathrm{E}}_{0},[\mathrm{keV}]$ & $\overline{\mathrm{E}}_{,}[\mathrm{keV}]$ & $\mathrm{E}_{\text {max }}[\mathrm{keV}]$ & $\begin{array}{c}\Delta \mathrm{E}=\mathrm{E}_{\mathrm{fin}}-\mathrm{E}_{\text {ini' }} \\
{[\mathrm{keV}]}\end{array}$ & $|\gamma|$ & $\left|\gamma_{\text {Cluster }}\right|$ \\
\hline \multirow{3}{*}{$\begin{array}{l}\text { D } \\
\text { (A) }\end{array}$} & $\mathrm{e}^{-}$ & 1 & 11 & 60 & 10 & - & 3.0 \\
\hline & $\mathrm{H}^{+}$ & 6 & 7 & 100 & 1 & - & 3.5 \\
\hline & $0^{+}$ & 12 & 13 & 200 & 1 & - & No data \\
\hline \multirow{3}{*}{$\begin{array}{l}\mathrm{D}+\mathrm{DFs} \\
\text { (B) }\end{array}$} & $\mathrm{e}^{-}$ & 1 & 12 & 150 & 11 & 4.7 & 3.5 \\
\hline & $\mathrm{H}^{+}$ & 6 & 8 & 300 & 2 & 2.0 & 2.5 \\
\hline & $\mathrm{O}^{+}$ & 12 & 15 & 450 & 3 & 3.2 & 2.8 \\
\hline \multirow{3}{*}{$\begin{array}{l}\text { D+DFs+Elf } \\
\text { (C) }\end{array}$} & $\mathrm{e}^{-}$ & 1 & 20 & 250 & 19 & 1.2 & 2.2 \\
\hline & $\mathrm{H}_{+}$ & 6 & 8 & 320 & 2 & 1.6 & 5.0 \\
\hline & $0^{+}$ & 12 & 15 & 500 & 3 & 4.5 & 4.0 \\
\hline
\end{tabular}

TABLE 1. Energetic characteristics of plasma particles before and after acceleration by (from top to bottom) : (A) a single dipolarization (labeled D), (B) dipolarization with DF bursts (labeled D+DFs), (C) like case B but followed by electric and magnetic fluctuations (labeled D+Dfs+Elf). The right column shows spectral indexes measured by Cluster on July 20, 2013 (01:33:08 - 01:48:11 UT).

anism (case A) can lead to the energy gain by one order of magnitude or slightly more for all types of particles. For protons and oxygen ions, the passage of successive DFs (case B) with duration less than 1 min leads to the resonant interactions and energy transfer from the field to the particles (Figure 4b). Here, electrons are less affected because of their small gyroperiods. In contrast, high-frequency electric and magnetic fluctuations following the passage of DFs (case C) can effectively lead to resonant energization of electrons (Figure 4c) with gain of energies about two orders of magnitude. In other words, combination of DFs passage and electric and magnetic fluctuations appears as the most effective mechanism of particle acceleration.

Table 1 summarizes energetic characteristics obtained for protons, oxygen ions and electrons, viz., initial average energy $\overline{\mathrm{E}}_{0}$; final average energy $\overline{\mathrm{E}}$, maximum energy $\mathrm{E}_{\max }$, net average energy gain $\Delta \mathrm{E}$, computed spectral index and spectral index obtained from in situ measurements. Note that the spectral index $\gamma$ is here calculated according to equation (6) of [Kronberg and Daly, 2013] for the following energy ranges: 70-95 keV for $\mathrm{e}^{-}, 90-160 \mathrm{keV}$ for $\mathrm{H}^{+}$, and $274-498 \mathrm{keV}$ for $0^{+}$.

In Table 1 , the smaller the value of $\gamma$ is, the larger the net particle energization. More specifically, in case B (i.e., dipolarization with DF bursts), the smallest $\gamma$ value is obtained for protons $\left(\gamma_{\mathrm{H}+}=2.0\right)$ while the largest value is observed for electrons $\gamma_{\mathrm{e}-}=4.7$, which reflects their less efficient energization as described above. In case C (dipolarization with DFs and electromagnetic fluctuations), a hardening (i.e., $\gamma$ decrease) of the electron energy spectra is obtained with $\gamma_{\mathrm{e}-}=1.2$.
No significant energization of $\mathrm{H}^{+}$and $\mathrm{O}^{+}$ions is noticeable in this case with $\gamma_{\mathrm{H+}}=1.6$ and $\gamma_{\mathrm{O}_{+}}=4.5 \mathrm{com}-$ pared to the case B.

The right column of Table 1 shows spectral indexes measured by the RAPID instrument onboard Cluster-4 on July 20, 2013 [Wilken et al., 2001]. Specifically, before the dipolarization onset (around 01:37:00 UT) the following spectral indices are observed for electrons $\left(\gamma_{0 \mathrm{e}}\right)$, protons $\left(\gamma_{0 \mathrm{p}}\right)$ and $0^{+}$ions $\left(\gamma_{00_{+}}\right): \gamma 0_{\mathrm{e}}=3.3 ; \gamma_{0 \mathrm{p}}=4.0$; $\gamma_{00+}=5.0$. Unfortunately, there are many gaps in the RAPID observations of energetic oxygen fluxes and the $\gamma$ index for oxygen ions at dipolarization onset cannot be calculated. As for protons and electrons, RAPID observations display a decrease of $\gamma$ near dipolarization onset (around 01:37:40 UT, case A). During the development of dipolarization, when the multiple DFs are observed A (around 01:39:50 UT, case B) protons and oxygen ions experience resonant acceleration and $\gamma$ indices accordingly decrease to minimum values. On the contrary, $\gamma_{e}$ does not decrease during this period. Equivalently, nonadiabatic electron energization does not operate during this time. At a later stage of dipolarization, when BZ stops increasing and highfrequency fluctuations are observed (after 01:47 UT, case C) the $\gamma_{e}$ decreases to its minimum value. At this time, no significant changes of $\gamma_{p}$ and $\gamma_{0}$ are noticeable. In summary, although $\gamma_{\mathrm{e}}$ values measured by Cluster and those derived from our numerical simulations cannot be compared one to one, they exhibit very similar time evolutions. The viewpoint developed in the present study is that these time evolutions closely reflect different sequences of resonant energization in the course of dipolarization. 


\section{CONCLUSION}

In this work, we focused on the effectiveness of particle energization during dipolarization events that have a complex multiscale character, being composed of successive dipolarization fronts followed by pile-up and consequent excitation of electric and magnetic fluctuations. We have shown that the multiscale changes of magnetic fields lead to the generation of induced electric fields that interact with plasma particles in a resonant manner, that is, the closer the time scale of the field variation to the particle gyroperiod, the more effective the transfer of energy from fields to particles. The three main time scales considered here are: (1) overall magnetic field dipolarization (of the order of a few minutes), (2) dipolarization fronts observed at smaller scales (typically, 20-30 s), (3) high-frequency fluctuations of electric and magnetic fields near electron cyclotron frequencies. It was shown that oxygen ions are more efficiently accelerated by the induced electric field due to the large scale dipolarization but are less sensitive than protons to DFs on the shorter time scales. Protons can be significantly accelerated by mechanisms (1) and (2) but the maximum net energy gain was found during DF passage. Electrons are less sensitive than ions to mechanisms (1) and (2) but are efficiently accelerated by the fast electric field fluctuations as summarized in the Figure 1.

Acknowledgements. Work by E.I.Z., H.V.M., E.E.G., V.Yu.P., E.A.K, P.W.D. was done in the frame of Volkswagen Foundation grant Az90 312. L.M.Z. acknowledges RFBR grants No.16-02-00479 and 16-52-16009 NCNILa..

\section{REFERENCES}

Angelopoulos, V., W. Baumjohann, C. F. Kennel, F. V. Coronti, M. G. Kivelson, R. Pellat, R. J. Walker, H. Luehr, G. Paschmann (1992). Bursty bulk flows in the inner central plasma sheet, J. Geophys. Res., 97, 4027-4039, doi:10.1029/91JA02701.

Angelopoulos, V., A. Runov, X. Z. Zhou, D. L. Turner, S. A. Kiehas, S.-S. Li, and 1. Shinohara (2013). Electromagnetic energy conversion at reconnection fronts, Science, 341, 1478-1482, doi:10.1126/science. 1236992.

Apatenkov, S. V., V. A. Sergeev, M. V. Kubyshkina, R. Nakamura, W. Baumjohann, A. Runov, 1. Alexeev, A. Fazakerley, H. Frey, S. Muhlbachler, P. W. Daly, J.A. Sauvaud, N.Ganushkina, T. Pulkkinen, G. D. Reeves, Y. Khotyaintsev (2007). Multi-spacecraft observation of plasma dipolarization/injection in the inner magnetosphere, Ann. Geophys., 25, 801-814, doi:10.5194/angeo-25-801-2007.

Artemyev, A. V., V. N. Lutsenko, and A. A. Petrukovich (2012). lon resonance acceleration by dipolarization fronts: Analytic theory and spacecraft observation, Ann. Geophys., 30, 317-324, doi:10.5194/angeo30-317-2012.

Ashour-Abdalla, M., G. Lapenta, R. J. Walker, M. ElAlaoui, H. Liang (2015). Multiscale study of electron energization during unsteady reconnection events, J. Geophys. Res., 120, 4784-4799, doi:10.1002/2014JA020316.

Baker, D. N., T. A. Fritz, R. L. McPherron, D. H. Fairfield, Y. Kamide, W. Baumjohann (1985). J. Geophys. Res., 90, 1205-1216, doi:10.1029/JA090iA02p01205.

Bauer, T. M., W. Baumjohann, R. A. Treumann, N. Sckopke, H. Luhr (1995). Low-frequency waves in the near-Earth plasma sheet, J. Geophys. Res., 100, 9605-9618, doi:10.1029/95JA00136.

Birn, J., M. F. Thomsen, M. Hesse (2004). Electron acceleration in the dynamic magnetotail: Test particle orbits in three-dimensional magnetohydrodynamic simulation fields, J. Plasma Phys., 11, 1825-1833, doi:10.1063/1.1704641.

Birn, J., A. V. Artemyev, D. N. Baker, M. Echim, M. Hoshino, L. M. Zelenyi, (2012). Particle Acceleration in the Magnetotail and Aurora, Space Sci. Rev., 173, 49-102, doi: 10.1007/s11214-012-9874-4.

Birn, J., M. Hesse, R. Nakamura, and S. Zaharia (2013), Particle acceleration in dipolarization events, J. Geophys. Res. Space Physics, 118, 1960-1971, doi:10.1002/jgra.50132.

Birn, J., A. Runov, and M. Hesse (2014). Energetic electrons in dipolarization events: Spatial properties and anisotropy, J. Geophys. Res. Space Physics, 119, 3604-3616, doi:10.1002/2013JA019738.

Birn, J., A. Runov, and M. Hesse (2015). Energetic ions in dipolarization events, J. Geophys. Res. Space Physics, 120, 7698-7717, doi:10.1002/2015JA021372.

Catapano, F., G. Zimbardo,S. Perri, A. Greco, A.V. Artemyev (2016). Proton and heavy ion acceleration by stochastic fluctuations in the Earth's magnetotail, Ann. Geophys., 34, 917-926, doi:10.5194/angeo34-917-2016.

Cattell, C. A., F. S. Mozer (1982). Electric fields measured by ISEE- 1 within and near the neutral sheet during quiet and active times, Geophys. Res. Lett., 9, 10411044, doi: 10.1029/GL009i009p01041.

Delcourt, D. C., A. Pedersen, and J.-A. Sauvaud, Dynamics of single-particle orbits during substorm expansion phase (1990). J. Geophys. Res., 95, 
20853-20865, doi: 10.1029/JA095iA12p20853.

Delcourt, D. C., Particle acceleration by inductive electric fields in the inner magnetosphere (2002). J. Atm. Solar Ter. Phys., 64, 551-559, doi:10.1016/S13646826(02)00012-3.

Drake, J. F., M. Swisdak, T. D. Phan, P. A. Cassak, M. A. Shay, S. T. Lepri, R. P. Lin, E. Quataert, and T. H. Zurbuchen (2009). lon heating resulting from pickup in magnetic reconnection exhausts, J. Geophys. Res., 114, A05111, doi:10.1029/2008JA013701.

El-Alaoui, M., R. L. Richard, M. Ashour-Abdalla, M. L. Goldstein, R. J. Walker (2013). Dipolarization and turbulence in the plasma sheet during a substorm: THEMIS observations and global MHD simulations, J. Geophys. Res. Space Physics, 118, 7752-7761, doi:10.1002/2013JA019322.

Fu, H. S., Y. V. Khotyaintsev, M. Andre, A. Vaivads (2011), Fermi and betatron acceleration of suprathermal electrons behind dipolarization fronts, Geophys. Res. Lett., 38, L16104, doi:10.1029/2011GL048528.

Galeev, A. A. (1979). The mechanism of magnetosphere substorms, Sov. Phys. Usp., 22, 196-197, doi:10.1070/PU1979v022n03ABEH005432.

Greco, A., A. Artemyev, G. Zimbardo (2015). Heavy ion acceleration at dipolarization fronts in planetary magnetotails, Geophys. Res. Lett., 42, 8280-8287, doi: 10.1002/2015GL066167.

Greco, A., S. Perri, G. Zimbardo, L. M. Zelenyi (2009). Particle acceleration by stochastic fluctuations and dawn-dusk electric field in the Earth's magnetotail, Adv. Space Res., 44, 528-533, doi:10.1016/j.asr.2009.05.012.

Grigorenko, E.E., M. Hoshino, M. Hirai, T. Mukai, L. M. Zelenyi (2009) "Geography" of ion acceleration in the magnetotail: X-line versus current sheet effects, J. Geophys. Res., 114, A03203, doi:10.1029/2008JA013811.

Grigorenko, E. E., L. M. Zelenyi, M. S. Dolgonosov, A. V. Artemiev, C. J. Owen, J. A. Sauvaud, M. Hoshino, M. Hirai (2011). Non-adiabatic lon Acceleration in the Earth Magnetotail and lts Various Manifestations in the Plasma Sheet Boundary Layer, Space Sci. Rev., 164, 133-181, doi:10.1007/s11214-011-9858-9.

Grigorenko, E. E.; A. Yu. Malykhin, E. A. Kronberg, Kh. V. , Malova, P. W. Daly (2015). Acceleration of ions to suprathermal energies by turbulence in the plasmoid-like magnetic structures, J. Geophys. Res. Space Physics, 120, 6541-6558, doi: 10.1002/2015JA021314.

Grigorenko, E. E., E. A. Kronberg,P. W. Daly, N. Yu. Ganushkina, B. Lavraud, J.-A. Sauvaud, L. M. Ze- lenyi (2016) . Origin of low proton-to-electron temperature ratio in the Earth's plasma sheet, J. Geophys. Res. Space Physics, 121, 9985-10,004, doi: 10.1002/2016JA022874.

Grigorenko, E. E., E. A. Kronberg, P. W. Daly (2017) . Heating and accleration of charged particles during magnetic dipolarizations, Cosmic Research, 55, 5766, doi: 10.1134/S0010952517010063.

Hamrin, M., P. Norqvist, T. Karlsson, H. Nilsson, H. S. Fu, S. Buchert, M. Andre, O. Marghitu, T. Pitkanen, B. Klecker, L. M. Kistler, 1. Dandouras (2013) . The evolution of flux pileup regions in the plasma sheet: Cluster observations, J. Geophys. Res. Space Physics, 118, 6279-6290, doi:10.1002/jgra.50603.

Harris, E. G. (1962) . On a Plasma sheath separating regions of oppositely directed magnetic fields, Nuovo Chimento., 23, 115-119, doi:10.1007/BF02733547.

Heyn, M. F, V. S. Semenov (1996) . Rapid reconnection in compressible plasma, J. Plasma Phys., 3, 2725-2741, doi:10.1063/1.871723.

Hoshino, M., A. Nishida, T. Yamamoto, S. Kokubun (1994) - Turbulent magnetic field in the distant magnetotail: Bottom-up process of plasmoid formation, Geophys. Lett., 21, 2 2935-2938, doi:10.1029/94GL02094.

Hoshino, M. (2005) . Electron surfing acceleration in magnetic reconnection, J. Geophys. Res.: Space Physics, 110, A10215, doi:10.1029/2005JA011229.

Ipavich, F. M., A. B. Galvin, G. Gloeckler, D. Hovestadt, B. Klecker, M. Scholer (1984) .Energetic (greater than $100 \mathrm{keV}) \mathrm{O}(+)$ ions in the plasma sheet, Geophys. Res. Lett., 11, 504-507, doi:10.1029/GL011i005p00504.

Keika, K., P. C. Brandt, S. Ohtani, D. G. Mitchell, K. Min, M. Nose, T. Obara, H. Koshiishi, H. Matsumoto (2010) .Mass-dependent evolution of energetic neutral atoms energy spectra during storm time substorms: Implication for $0+$ nonadiabatic acceleration, J. Geophys. Res., 115, A00112, doi:10.1029/2010JA015889.

Kronberg, E. A. and Daly, P. W. (2013). Spectral analysis for wide energy channels, Geosci. Instrum. Method. Data Syst., 2, 257-261, doi:https://doi.org/10.5194/gi-2-257-2013.

Kronberg, E. A., E. E. Grigorenko, D. L. Turner, P. W. Daly, Y. Khotyaintsev, L. Kozak (2017) . Comparing and contrasting dispersionless injections at geosynchronous orbit during a substorm event, J. Geophys. Res., 122, 3055-3072, doi:10.1002/2016JA023551.

Lembege, B., R. Pellat (1982) . Stability of a thick two-dimensional quasineutral sheet, Phys. Fluids, 25 , doi:http://dx.doi.org/10.1063/1.863677. 
Liang, H., G. Lapenta, R. J. Walker, D. Schriver, M. ElAlaoui, J. Berchem (2017) . Oxygen acceleration in magnetotail reconnection, J. Geophys. Res., 122, 618-639, doi:10.1002/2016JA023060.

Longcope, D. W., E. R. Priest (2007). Fast magnetosonic waves launched by transient, current sheet reconnection, J. Plasma Phys., 14, 122905, 11, doi:10.1063/1.2823023.

Lu, S., A. V. Artemyev, V. Angelopoulos, Q. Lu, and J. Liu (2016), On the current density reduction ahead of dipolarization fronts, J. Geophys. Res. Space Physics, 121, 4269-4278, doi:10.1002/ 2016JA022754.

Lui, A. T. Y. (2014) . Evidence for two types of dipolarization in the earth's magnetotail, J. Atm.Sol. Terr. Phys., 115, 17-24, doi:10.1016/j.jastp.2013.10.002.

Nakamura, R., W. Baumjohann, B. Klecker, Y. Bogdanova, A. Balogh, H. Reme,; J. M. Bosqued, 1. Dandouras, J. A. Sauvaud, K.H. Glassmeier, L. Kistler, C. Mouikis, T. L. Zhang, H. Eichelberger, A. Runov (2002). Motion of the dipolarization front during a flow burst event observed by Cluster, Geophys. Res. Lett., 29, 3-1, 1942, doi:10.1029/2002GL015763.

Nakamura, R., W. Baumjohann, M. Fujimoto, Y. Asano, A. Runov, C. J. Owen, A. N. Fazakerley, B. Klecker, H. ReMe, E. A. Lucek, M. Andre, Y. Khotyaintsev (2008). Cluster observations of an ion-scale current sheet in the magnetotail under the presence of a guide field, J. Geophys. Res. Space Phys., 113, A07S16, doi:10.1029/2007JA012760.

Nosé, M., S. Ohtani, A. T. Y. Lui, S. P. Christon, R. W. McEntire, D. J. Williams, T. Mukai, Y. Saito, K. Yumoto (2000). Change of energetic ion composition in the plasma sheet during substorms, J. Geophys. Res., 105, 23277-23286, doi:10.1029/2000JA000129.

Ono, Y., M. Nose, S. P. Christon, A. T. Y. Lui (2009). The role of magnetic field fluctuations in nonadiabatic acceleration of ions during dipolarization, J. Geophys. Res., 114, A05209, doi:10.1029/2008JA013918.

Perri, S., F. Lepreti, V. Carbone, A. Vulpiani (2009). Dynamical properties of test particles in stochastic electromagnetic fields, Communications in Nonlinear Science and Numerical Simulation, 14, 23472352, doi:10.1016/j.cnsns.2008.06.023.

Retino, A., R. Nakamura, A. Vaivads, Y. Khotyaintsev, T. Hayakawa, K. Tanaka, S. Kasahara, M. Fujimoto, 1. Shinohara, J. P. Eastwood, M. Andre, W. Baumjohann, P. W. Daly, E. A. Kronberg, N. CornilleauWehrlin (2008). Cluster observations of energetic electrons and electromagnetic fields within a reconnecting thin current sheet in the Earth's mag- netotail, J. Geophys. Res. Space Physics, 113, A12215, doi:10.1029/2008JA013511.

Runov, A., V. Angelopoulos, V. A. Sergeev, K.H. Glassmeier, U. Auster, J. McFadden, D. Larson, 1. Mann (2009). Global properties of magnetotail current sheet flapping: THEMIS perspectives, Ann. Geophys, 27, 319-328, doi: http://www.ann-geophys.net/27/319/2009/.

Runov, A., V. Angelopoulos, M. Sitnov, V. A. Sergeev, R. Nakamura,Y. Nishimura, H. U. Frey, J. P. McFadden, D. Larson, J. Bonnell, K.H. Glassmeier, U. Auster, M. Connors, C. T. Russell, H. J. Singer (2011). Dipolarization fronts in the magnetotail plasma sheet, Planetary and Space Science, 59, 517-525, doi: 10.1016/j.pss.2010.06.006.

Runov, A., V. Angelopoulos, X.Z. Zhou, X.J. Zhang, S. Li, F. Plaschke, J. A. Bonnell (2011). THEMIS multicase study of dipolarization fronts in the magnetotail plasma sheet, J. Geophys. Res., 116, A05216, doi:10.1029/2010JA016316.

Runov, A., V. A. Sergeev, V. Angelopoulos, K.H. Glassmeier, H. J. Singer (2014). J. Geophys. Res. Space Phys., 119, 1643-1657, doi:10.1002/2013JA019384.

Semenov, V. S., T. Penz, V. V. Ivanova, V. A. Sergeev, H. K. Biernat, R. Nakamura, M. F. Heyn, 1. V. Kubyshkin, 1. B. Ivanov (2005). Reconstruction of the reconnection rate from Cluster measurements: First results, J. Geophys. Res. Space Phys., 110, A11217, doi:10.1029/2005JA011181.

Sergeev, V. A., R. C. Elphic, F. S. Mozer, A. Saint-Marc, J. A. Sauvaud (1992). A two satellite study of nightside flux transfer events in the plasma sheet, Planetary and Space Science, 40, 1551-1572, doi:10.1016/0032-0633(92)90052-P.

Sergeev, V., V. Angelopoulos, S. Apatenkov, J. Bonnell, R. Ergun, R. Nakamura, J. McFadden, D. Larson, A. Runov (2009). Kinetic structure of the sharp injection/dipolarization front in the flow-braking region, Geophys. Res. Lett., 36, L21105, doi:10.1029/2009GL040658.

Sergeev, V., V. Angelopoulos, M. Kubyshkina, E. Donovan, X.Z. Zhou, A. Runov, H. Singer, J. McFadden, R. Nakamura (2011). Substorm growth and expansion onset as observed with ideal ground-spacecraft THEMIS coverage, J. Geophys. Res., 116, A00126, doi:10.1029/2010JA015689.

Sitnov, M. 1., M. Swisdak, A. V. Divin (2009) .Dipolarization fronts as a signature of transient reconnection in the magnetotail, J. Geophys. Res., 114, A04202, doi: 10.1029/2008JA013980.

Sitnov, M. 1., M. Swisdak (2011). Onset of collisionless magnetic reconnection in two-dimensional current 
sheets and formation of dipolarization fronts, J. Geophys. Res., 116, A12216, doi:10.1029/2011JA016920.

Sharma A. S., Nakamura R., Runov A., Grigorenko E.E., Hasegawa H., Hoshino M., Louarn P., Owen C.J., Petrukovich A., Sauvaud J.-A., Semenov V.S., Sergeev V.A., Slavin J.A., Sonnerup B.U.O., Zelenyi L.M., Fruit G., Haaland S., Malova H., Snekvik K. (2008) . Transient and Localized Processes in the Magnetotail, Rev. Ann. Geophys., 26, 1-51, doi: 10.5194/angeo-26-955-2008.

Slavin, J. A., C. J. Owen, M. W. Dunlop, E. Boralv, M. B. Moldwin, D. G. Sibeck, E. Tanskanen, M. L. Goldstein, A. Fazakerley, A. Balogh, E. Lucek, 1. Richter, H. Reme, J. M. Bosqued (2003) .Cluster four spacecraft measurements of small traveling compression regions in the near-tail, doi:10.1029/2003GL018438.

Ukhorskiy, A. Y., M. 1. Sitnov, V. G. Merkin, M. Gkioulidou,D. G. Mitchell (2017) . lon acceleration at dipolarization fronts in the inner magnetosphere, J. Geophys. Res. Space Physics, 122, 3040-3054, doi:10.1002/2016JA023304.

Veltri, P., G. Zimbardo, A. L. Taktakishvili, L. M. Zelenyi (1998) . Effect of magnetic turbulence on the ion dynamics in the distant magnetotail, J. Geophys. Res., 103, 14897-14916, doi:10.1029/98JA00211.

Yamada, M., R. Kulsrud, H. Ji, Magnetic reconnection (2010) . Rev. Mod. Phys., 82, 603-664, doi:10.1103/RevModPhys.82.603.

Yao, Z., A. N. Fazakerley, A. Varsani, I. J. Rae, C. J. Owen, D. Pokhotelov, C. Forsyth, R. L. Guo, S. C. Bai, S. T. Yao, N. Doss (2016) . Substructures within a dipolarization front revealed by high-temporal resolution Cluster observations, J. Geophys. Res. Space Physics, 121, 5185-5202, doi:10.1002/2015JA022238.

Zelenyi, L. M., J. G. Lominadze, A. L. Taktakishvili (1990). Generation of the energetic proton and electron bursts in planetary magnetotails, J. Geophys. Res., 95, 3883-3891, doi:10.1029/JA095iA04p03883.

Zelenyi, L.M., V. A. Milovanov (2004) . Fractal topology and strange kinetics: from percolation theory to problems in cosmic electrodynamics, Phys. Usp., 47, R01, doi:10.1070/PU2004v047n08ABEH001705.

Zelenyi L. M., M. Oka, H. V. Malova, M. Fujimoto, D. Delcourt, W. Baumjohann (2007) . Particle Acceleration in Mercury's Magnetosphere, Space Sci. Rev., doi:10.1007/s11214-007-91693.

Zelenyi, L.M, A. V. Artemyev, H. V. Malova and V. Yu. Popov (2008) . Marginal stability of thin current sheets in the Earth's magnetotail, J. Atmos. Sol. Terr.
Phys., 70, 325-333.

Zelenyi, L. M., A. V. Artemyev, H. V. Malova, A. V. Milovanov, G. Zimbardo (2008) . Particle transport and acceleration in a time-varying electromagnetic field with a multi-scale structure, Phys. Lett., 372, 62846287, doi:10.1016/j.physleta.2008.08.035.

Zelenyi, L. M., A. G. Korgov, H. V. Malova, V. Yu. Popov, A. V. Artemyev and D. C. Delcourt (2010) . Charged particle acceleration in the Hermean magnetosphere: the role of dipolarizations, plasma turbulence and induction electric fields, Adv. Geoscienc., 9-28.

Zelenyi, L.M., H.V. Malova, A.V. Artemyev, V.Yu. Popov and A.A.Petrukovich (2011) . Thin current sheets in collisionless plasma: equilibrium structure, plasma instabilities, and particle acceleration, Plasma Phys. Rep., 2011, 37, 118-160, doi: https://doi.org/10.1134/S1063780X1102005X

Zimbardo, G., A. Greco, P. Veltri, A. L. Taktakishvili and L. M. Zelenyi (2004) . Double peak structure and diamagnetic wings of the magnetotail current sheet, Ann. Geophys., 22, 2541-2546, doi: https://hal.archives-ouvertes.fr/hal-00317515.

Zhou, X.Z., V. Angelopoulos, V. A. Sergeev, and A. Runov (2010). Accelerated ions ahead of earthward propagating dipolarization fronts, J. Geophys. Res., 115, A00103, doi:10.1029/2010JA015481.

Zhou, X.Z., Y. S. Ge, V. Angelopoulos, A. Runov, J. Liang, X. Xing, J. Raeder, Q.G. Zong (2012). Dipolarization fronts and associated auroral activities: 2. Acceleration of ions and their subsequent behavior, J. Geophys. Res., 117, A10227, doi:10.1029/2012JA017677.

*CORRESPONDING AUTHOR: H. V. MALOVA,

Nuclear Physics Institute, Moscow State University,

Moscow, Russia

email: hmalova@yandex.ru

(C) 2018 the Istituto Nazionale di Geofisica e Vulcanologia. All rights reserved. 